\title{
Fabrication of Tilted Fiber Bragg Grating as A Sensor of Refractive Index of Liquids
}

\author{
Akihiro KAMEYAMA, ${ }^{1}$ Eka MAULANA,,${ }^{1, \dagger}$ Masahito KATTO, ${ }^{2}$ and Atsushi YOKOTANI ${ }^{1}$ \\ ${ }^{1}$ Faculty of Engineering, University of Miyazaki, Miyazaki 889-2192 \\ ${ }^{2}$ Center for Collaborative Research and Community Cooperation, University of Miyazaki, Miyazaki 889-2192
}

(Received July 11, 2012)

\begin{abstract}
We demonstrated that a simple technique combining a 266-nm laser and a phase mask was quite effective at fabricating tilted fiber Bragg gratings (TFBG) for refractive index sensor of liquids. Using fabricated TFBGs that have the tilted angles of $3.3,6.7,7.3,8.0$, and $9.9^{\circ}$, we tried to measure the refractive index of liquids that have different indices. We have directed our attention to the fact that the wavelength of cladding mode becomes longer as the refractive index of sample liquids increases. Utilizing this wavelength shift, we proposed a new measurement method. As a result, we could successfully measure the refractive index of liquids within a range from 1.00 to 1.41 with a maximum resolution of $2.1 \times 10^{-3}$. In addition, we have found that a writing length only $2 \mathrm{~mm}$ long is enough for measuring the refractive index of liquid.
\end{abstract}

Key Words: Tilted fiber bragg grating, Refractive index, Phase mask, Cladding mode

\section{Introduction}

Fiber sensors are widely used in the sensing of physical properties such as temperature, strain, vibration, pressure, liquid level, and so on. ${ }^{1)}$ The increase in fiber sensor development for these purposes has many advantages, i.e., electromagnetic immunity, small size, stability, harmlessness, and high sensitivity. ${ }^{2)}$ Photosensitivity of fiber core was reported by Hill et al. in 1978. ${ }^{3)}$ Since then, this invention has been a significant background for the many kinds of fiber sensor developments. The fiber Bragg gratings (FBGs), which consist of a periodic modulation of the refractive index in the core of a single mode optical fiber, have especially become widely used for distortion sensors. ${ }^{4}$

Recently, in addition to the FBG sensors, tilted fiber Bragg grating (TFBG) has become to attract considerable attention for sensing applications. The information in the cladding as well as the properties of the core affects the reflection spectrum in TFBG. Therefore, in TFBG, non-mechanical phenomena, which are information through the surface of the cladding, can be detected, while normal FBG can observe only mechanical phenomena in the core, such as change in length, since the cladding prevents the optical information coming to the core from the outside. Although one might think that the temperature can be detected using the normal FBG at a glance, this is also measured by mechanical volume change due to the thermal expansion. TFBG has been conventionally fabricated using the Lloyd mirror interferometer by a $244-\mathrm{nm} \mathrm{Ar}^{+}$ion laser, and the spectra have been observed in previous work. ${ }^{5)}$ In addition, it has been reported that the refractive index of liquid can be detected in principle by using the envelope of the cladding mode of TFBG. However, adjusting the Lloyd mirror causes an instability of the period of the grating, and furthermore, using the $\mathrm{Ar}^{+}$ion laser results in a large difficulty when this technique is considered for application on a commercial basis.

In this research, we have developed a simplified fabrication technique for TFBG using a combination of a 266-nm laser and a conventional phase mask that can be used without a complicate optical adjustment. Moreover, we also tried to characterize the fabricated TFBG as a sensor for the refractive index of liquids. As a result, we found a new method to estimate the index of liquid with a wider measurement range compared to the conventional method that has been reported in previous work.

\section{Basic properties of TFBG}

The FBGs are made using a laser beam interference technique. Fundamental structures of FBG and TFBG are shown in Fig. 1. A single core mode is produced in the FBG transmission spectrum. Basically, the wavelength shift in the core mode is used to detect mechanical change in the fiber. Therefore, the wavelength of core mode is utilized to measure only physical parameters. ${ }^{6)}$

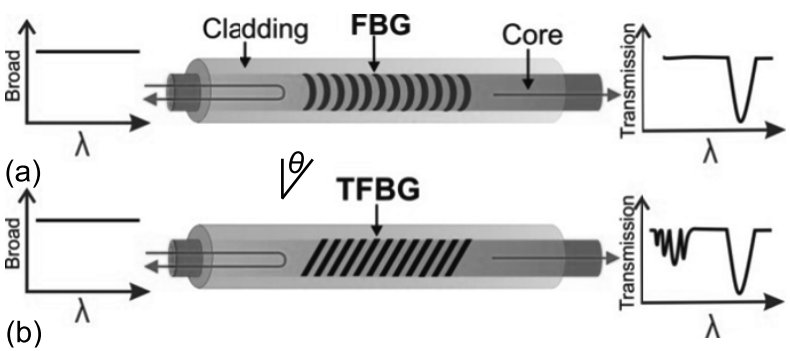

Fig. 1 Structure of (a) FBG and (b) TFBG.

${ }^{\dagger}$ Faculty of Engineering, University of Brawijaya, JI. Veteran Malang 65145, Indonesia 
The wavelength of core mode in the FBG $\left(\lambda_{B}\right)$ can be calculated by the following equation,

$$
\lambda_{B}=2 n_{\text {eff.core }} \Lambda,
$$

where $n_{\text {eff.core }}$ is the effective refractive index of the core, and $\Lambda$ is the grating period.

FBGs are intrinsically insensitive to the environment, because the core modes are well-screened from incident of the light from outside due to the presence of the cladding. ${ }^{7)}$ On the other hand, in the case of the TFBG that has a tilt angle $\theta$, not only the core mode but also a number of cladding modes are observed in the reflection spectrum. The reflection wavelengths of the cladding modes $\left(\boldsymbol{\lambda}_{\text {cladding }}\right)$ are calculated using the following equation,

$$
\lambda_{\text {cladding }}=\frac{\left(n_{\text {eff.core }}+n_{\text {eff.cladding }}\right) \Lambda}{\cos \theta},
$$

where $n_{\text {eff.cladding }}$ is the effective refractive index of the cladding that corresponds to each reflecting order in the cladding mode. The wavelength and amplitude of the cladding mode are affected by optical properties of the surrounding material. The detailed spectral behaviors in the core and the cladding modes have been reported in previous work. ${ }^{8}$

\section{Experimental}

\subsection{Fabrication of TFBG}

The experimental setup for fabrication of TFBG is shown in Fig. 2. We used a $4 \omega \mathrm{Nd}$ :YAG laser and a wavelength converter to produce a pulsed 266-nm UV laser beam. The beam was reflected by five mirrors and linearly focused on a sample fiber by three cylindrical lenses. This laser produced laser pulses with an energy of $50 \mathrm{~mJ} /$ pulse. The beam was introduced to an optical fiber core through a phase mask made of silica glass. In this technique, a periodically modulated UV beam was produced by interference of two diffracted laser beams due to the phase mask. The minimum distance between the phase mask and the fiber sample was approximately $1 \mathrm{~mm}$. Tension of the fiber was kept at $5.9 \times 10^{-4} \mathrm{~N}$ during fabrication process with the UV beam irradiation.

A $\mathrm{H}_{2}$ loaded SBG-15 (Newport corp.) photosensitive optical fiber was used for the sample fiber. This fiber is a single mode and germanium-boron-codoped fiber. We used 250-mm-long sample fibers. A 50-mm-long polymer jacket of the center part of the fiber was stripped, in order to expose the fiber core with UV beam irradiation. 20-mm-long polymer

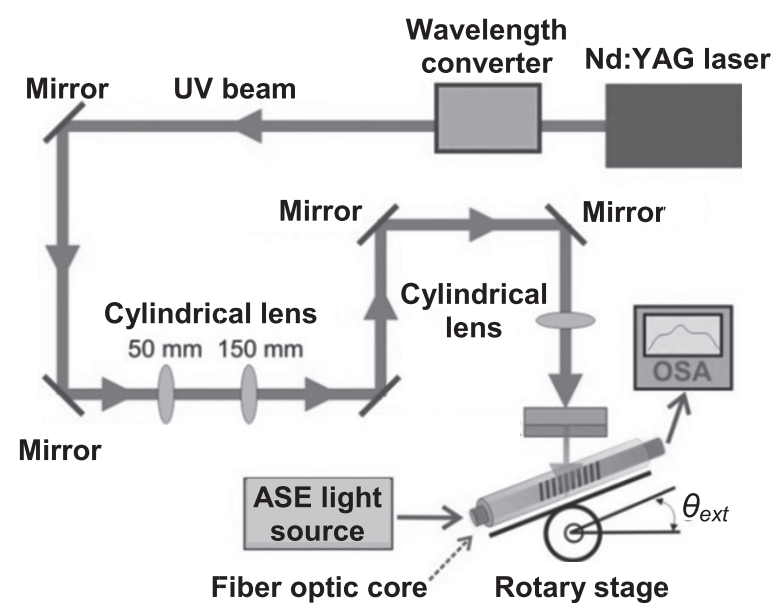

Fig. 2 Experimental setup. jackets at the both ends of the fiber were also stripped, in order to connect an optical spectrum analyzer (OSA) and an ASE light source. The fiber Bragg grating was made in the center part of fiber using a phase mask technique. A phase mask with a grating period of $1.065 \mu \mathrm{m}$ was used. This phase mask creates a grating in the fiber core with a period $\Lambda_{t f b s}$ along the longitudinal direction, which follows equation 3 .

$$
\Lambda_{t f b g}=\frac{\Lambda_{p m}}{2 \cos \theta_{e x t}},
$$

where $\Lambda_{p m}$ and $\theta_{e x t}$ are grating period of the phase mask and the external tilt angle between the grating and the sample fiber, respectively. A 10-mm-long phase mask was used in this experiment

The fiber was fixed on a rotary stage to adjust the $\theta_{\text {ext }}$ easily. The $\theta_{\text {ext }}$ were chosen at $5,10,11,12$, and $15^{\circ}$, which corresponded to the incident angles of the modulated beam to the fiber surface. Since the UV beam refracts between air and fiber material during fabrication, the tilt angles in the fiber core become $3.3,6.7,7.3,8.0$, and $9.9^{\circ}$, respectively. The probe light with a wavelength range from 1520 to $1610 \mathrm{~nm}$ was used for the transmission spectral measurement. Two mechanical splicers were used for connecting the fiber to the light source and the OSA. The typical irradiation period of the UV beam to obtain the enough intensity for measurement was 20 minutes. We checked the spectral change during TFBG fabrication.

\subsection{Refractive index measurement}

We applied a droplet of sample liquid that covered the whole 10-mm-long TFBG for refractive index measurement. Experimental setup to measure the refractive index of liquid is shown in Fig. 3. Two holders were used to keep the TFBG stable on the glass plate without applying tension. The liquids used included water, ethanol, and glycerine solutions. The reason for choosing the glycerine solution is that the refractive index of the solution can be easily adjusted in a wide index range by simply mixing the glycerine and water. ${ }^{9)}$ The concentration of glycerine solution used in the experiment was from $12 \%$ to $84 \%$. The detailed information for the index of samples is summarized in Table 1.

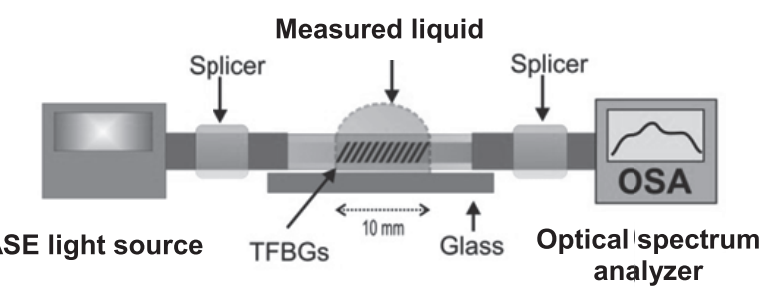

Fig. 3 Refractive index measurement procedure.

Table 1 Refractive index of sample.

\begin{tabular}{lc}
\hline \multicolumn{1}{c}{ Sample } & Ref. index \\
\hline Air & 1.0003 \\
\hline Water & 1.3255 \\
\hline Ethanol & 1.3539 \\
\hline Glycerine 12\% & 1.3417 \\
\hline Glycerine 24\% & 1.3579 \\
\hline Glycerine 35\% & 1.3727 \\
\hline Glycerine 46\% & 1.3876 \\
\hline Glycerine 66\% & 1.4146 \\
\hline Glycerine 84\% & 1.4389
\end{tabular}

The Review of Laser Engineering November 2012 
We observed the core and the cladding modes in the transmission spectra and investigated the relationship between the spectral change and the change in refractive index.

\section{Results}

\subsection{Characteristic of fabricated TFBG}

Transmittance spectra of $0^{\circ}$-TFBG (namely, normal FBG) after 2 minutes irradiation are shown in Fig. 4. Only the core mode $\mathrm{LP}_{01}$ was observed in this spectrum. The intensity of the core mode $\mathrm{LP}_{01}$ was $9.5 \mathrm{~dB}$ at $1544 \mathrm{~nm}$.

Figure 5 (a) shows the transmission spectra of the $6.7^{\circ}$ TFBG after 20 minutes irradiation time. We investigated not only core mode $\mathrm{LP}_{01}$, but also the cladding modes in a spectral range approximately from 1520 to $1563 \mathrm{~nm}$. The core mode shifted to the longer wavelength and reached $1565 \mathrm{~nm}$, and its intensity decreased to $2.5 \mathrm{~dB}$. The maximum intensity of $9 \mathrm{~dB}$ in the cladding mode was observed at a wavelength of $1546 \mathrm{~nm}$. In the cladding mode, superposition of two coupling modes $\left(\mathrm{LP}_{0 \mathrm{n}}\right.$ and $\left.\mathrm{LP}_{1 \mathrm{n}}\right)$ was observed.

Figure 5 (b) shows the transmission spectra of the $8.0^{\circ}$ TFBG after 20 minutes irradiation. In the case of $8.0^{\circ}$-TFBG, the longer wavelength and smaller intensity of the core mode compared with $6.7^{\circ}$-TFBG were observed. The peak wavelength and the intensity were $1580 \mathrm{~nm}$ and $2 \mathrm{~dB}$, respectively. The cladding modes were observed in a range from 1520 to $1578 \mathrm{~nm}$. The maximum intensity of the cladding mode was $11.5 \mathrm{~dB}$ at $1548 \mathrm{~nm}$. We observed two coupling modes in the cladding mode as well.

Figure 5 (c) shows the transmission spectra of the $9.9^{\circ}$ TFBG after 20 minutes irradiation. In this case, the core mode disappeared. The cladding modes were observed in a range from 1520 to $1580 \mathrm{~nm}$. Although the apparent superposition of the two coupling modes was not observed, we confirmed that both were present because the wavelength difference between two adjacent peaks was almost the same as that in the case of $8.0^{\circ}$-TFBG.

\subsection{Characteristic of liquid measurement}

By using the $8.0^{\circ}$-TFBG, which exhibited the strongest coupling in the cladding mode, the experiments for measuring the refractive index of liquids were performed. The results are shown in Fig. 6.

In Fig. 6, spectral change of a selected peak in the cladding modes is indicated. We selected a peak at $1543.76 \mathrm{~nm}$, which was nearly the strongest peak in the air. As shown in the figure, the peak wavelength became longer as the refractive index of liquid became larger. As a result, the wavelength shift-

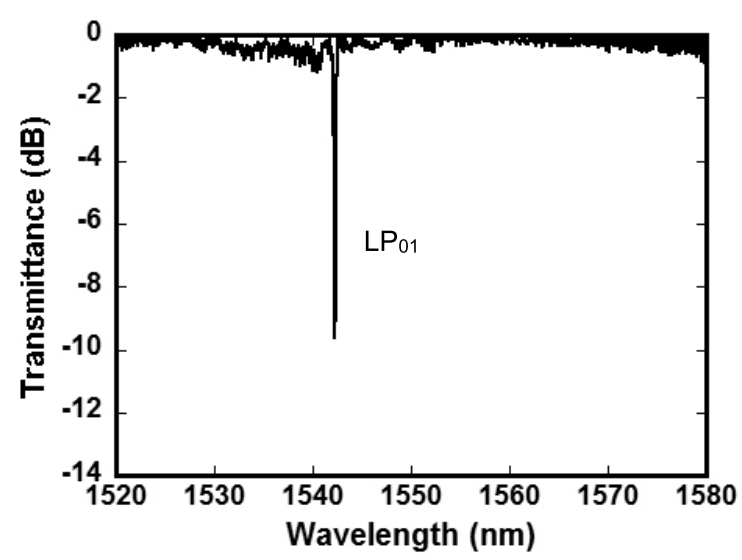

Fig. 4 Transmission spectra $0^{\circ}$-TFBG.
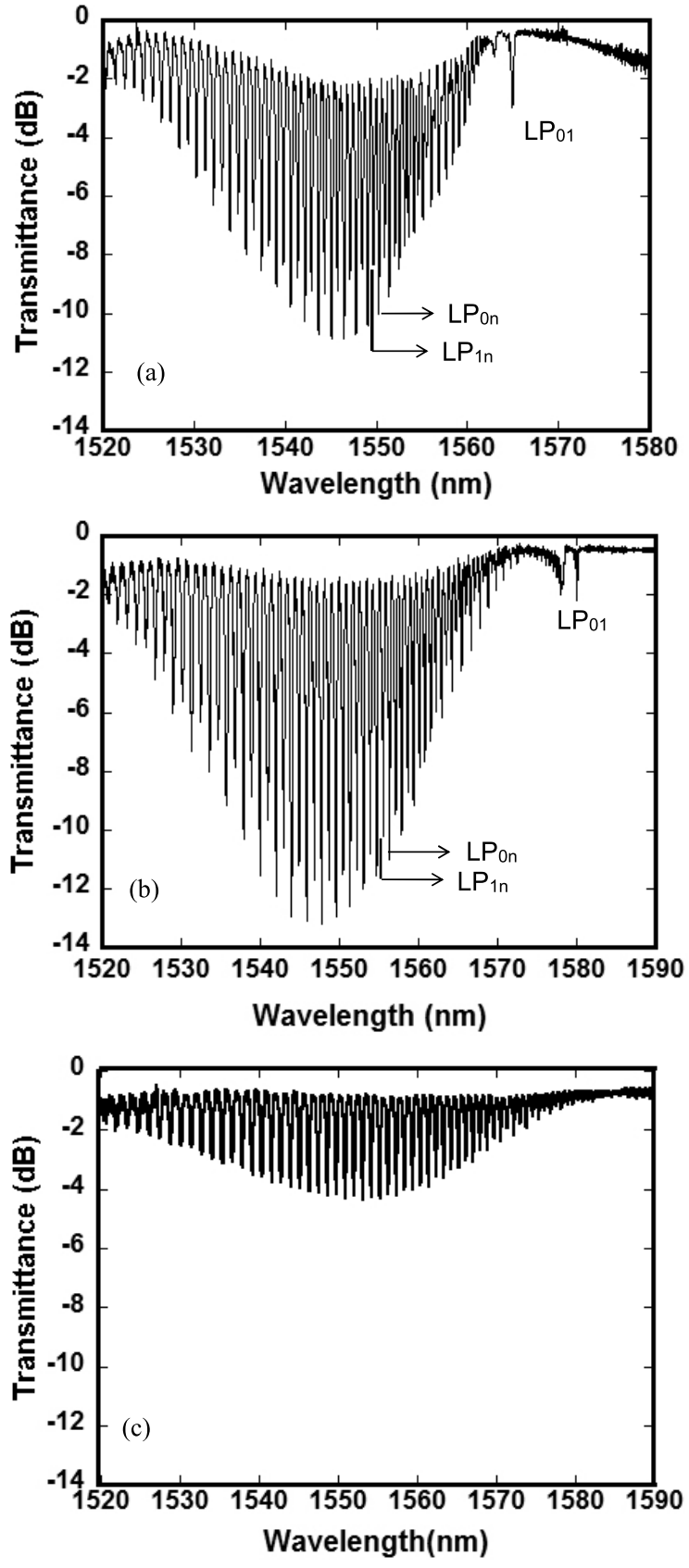

Fig. 5 Transmission spectra of fabricated TFBGs with tilted angles of (a) $6.7^{\circ}$, (b) $8.0^{\circ}$ and (c) $9.9^{\circ}$.

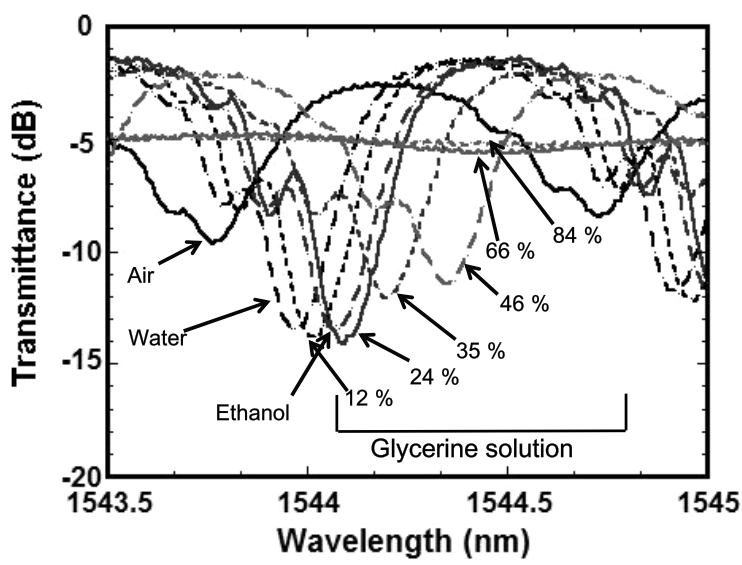

Fig. 6 Refractive index measurement of liquids in $8.0^{\circ}$ TFBG. 
ed from 1543.76 to $1544.40 \mathrm{~nm}$, corresponding to the index from 1.00 to 1.41 . In addition to the wavelength change, the smooth change in the peak intensity was also observed. Laffont $e t a l .^{5)}$ used this change in intensity to estimate the index of liquid and demonstrated that the refractive index of liquids from 1.35 to 1.44 was able to be estimated by calculating areas of the envelope of cladding modes. In this work, we concentrated on investigating the change in wavelength, intending a more accurate measurement.

Similar experiments have been done for the other TFBGs with different tilt angles. Figure 7 shows the relative wavelength shift to the refractive index of the liquids and air for TFBGs that have various tilted angles. The smooth lines in Fig. 7 show the relative wavelength shift function $f(n)$ to the refractive index $n_{l}$ of liquids that was increased exponentially by optimized approximation calculation,

$$
f(n)=A\left(e^{B(n l-1)}-1\right),
$$

The maximum correlation was achieved at $8.0^{\circ}$-TFBG. From the tilt angle of 3.3 to $8.0^{\circ}$, the correlation between the index and obtained data became stronger as the tilt angle became larger. However, in the case of $9.9^{\circ}$-TFBG, the correlation became slightly weaker than at $8.0^{\circ}$-TFBG.

We estimated the resolution of index change $\Delta n$ in this method with the following equation,

$$
\Delta n=\frac{d n_{l}}{d \Delta \lambda},
$$

where $n_{l}$ is the index of liquids and $\Delta \lambda$ is the relative wavelength shift. Figure 8 shows the results. The resolution indi-

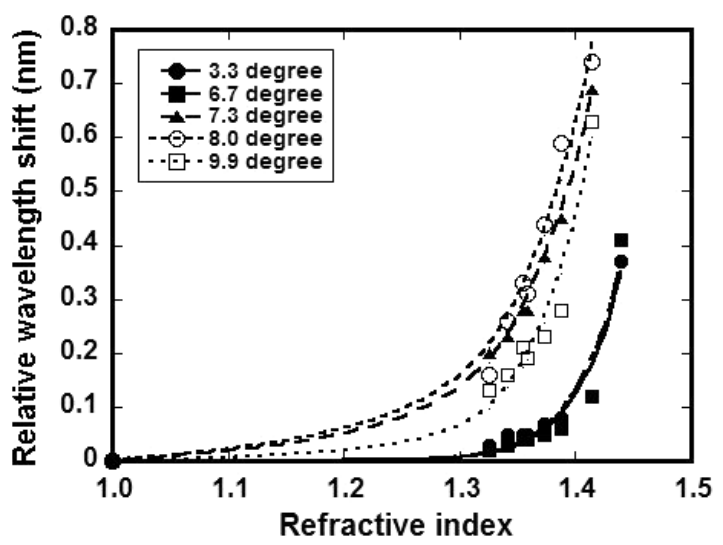

Fig. 7 Relative wavelength shift of TFBG with different tilt angle.

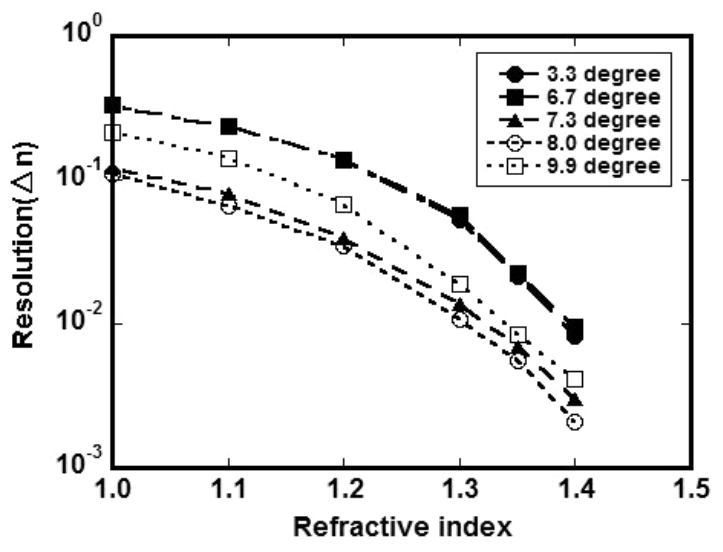

Fig. 8 Resolution of refractive index. cated in Fig. 8 was obtained as the index change corresponding to the spectral change of $20 \mathrm{pm}$ since the wavelength resolution of the OSA used in this experiment was $20 \mathrm{pm}$. As shown in the figure, $8.0^{\circ}$-TFBG indicated the maximum sensitivity within a wide refractive index range. The values were approximately from $1.1 \times 10^{-1}$ to $2.1 \times 10^{-3}$ depending on the index.

The relative wavelength shift of the $8.0^{\circ}$-TFBG with the different writing lengths is shown in Fig. 9. The relative wavelength shifts of the 5- and 2-mm long $8.0^{\circ}$-TFBG are the same as that of the $10-\mathrm{mm}$-long $8.0^{\circ}$-TFBG. This fact implies that the writing length of $2 \mathrm{~mm}$ is enough to obtain information from the liquid that is almost the same as the data mentioned above.

\section{Discussion}

Based on our experiment, we can say that TFBG was successfully fabricated by combining the 266-nm laser and the phase mask. This technique is more simple compared to the previous method ${ }^{5)}$ due to a combination of the 244-nm laser and the Lloyd mirror interferometer. The maximum peak intensity in cladding mode and sensitivity were achieved in the case $8.0^{\circ}$-TFBG. The intensity of $9.9^{\circ}$-TFBG was weaker than that of $8.0^{\circ}$-TFBG. The reason for this is considered to be some degradations of interference due to the increase in distance between the phase mask and the fiber sample position during the fabrication.

Figure 10 shows the peak wavelength of $8.0^{\circ}$-TFBG as a function of the refractive index of liquids. The square dots show the measured peak wavelength with $8.0^{\circ}$-TFBG. The

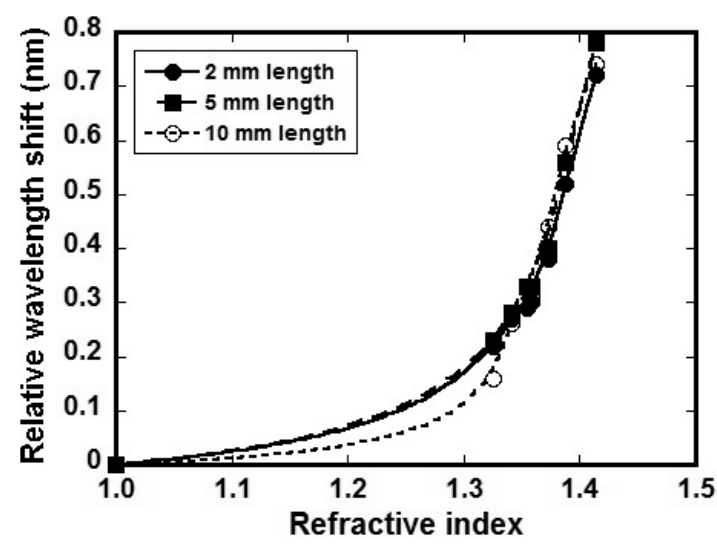

Fig. 9 Relative wavelength shift of $8.0^{\circ}$-TFBG with the different writing lengths.

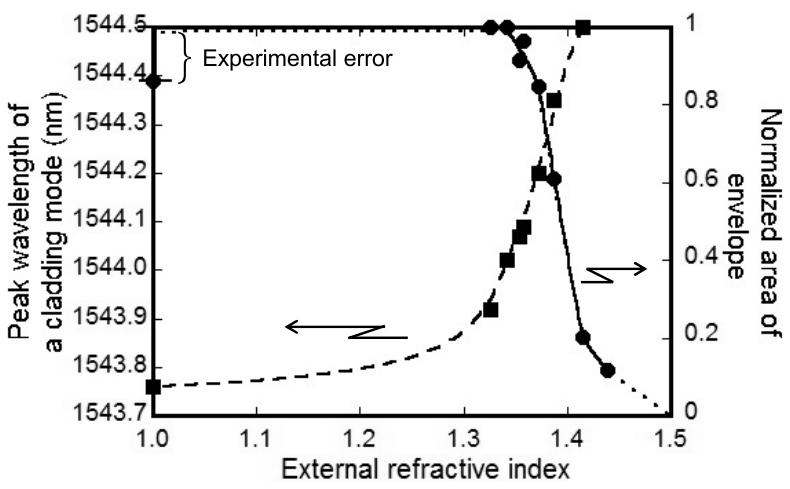

Fig. 10 Peak wavelength compared by Laffont's method in $8.0^{\circ}$-TFBG. 
circle dots in the figure indicate the data treated with the method presented by Laffont et al.. ${ }^{5)}$ By comparing these two curves, we can say that our estimation method has the wider measurement range. In principle, it is considered to be very difficult to estimate the index less than 1.33 by their method.

Finally, we could demonstrate that the writing length of only $2 \mathrm{~mm}$ was necessary to measure the refractive index of liquid as shown in Fig. 9. This fact suggests the possibility for development of very small sensors which may be applied in medical and biological applications.

\section{Conclusions}

Several tilted fiber Bragg gratings (TFBGs) have been fabricated, and its transmittance spectra during fabrication have been investigated. We have also measured the refractive index of liquid using the response of wavelength shift in a cladding mode against the index change of a liquid droplet. Based on our experiment, we conclude that:

1) TFBG can be fabricated with a simple technique combining a 266-nm laser and the phase mask.

2) The fabricated $8.0^{\circ}$-TFBG can be used to measure the refractive index of liquid from 1.00 to 1.41 .
3) The resolution of detecting the index change is $1.1 \times$ $10^{-1}$ to $2.1 \times 10^{-3}$ for $8.0^{\circ}$-TFBG depending on the index.

4) It has been found that TFBG only $2 \mathrm{~mm}$ long is enough to measure the refractive index of liquid.

The TFBG is considered to be very promising for the sensors of bio-chemical phenomena in medical and biological fields.

\section{References}

1) X. Dong, H. Zhang, B. Liu, and Y. Miao: Photonic Sensors 1 (2011) 6.

2) S. Yin, P. B. Ruffin, and F. T. S. Yu: Fiber Optic Sensors. Second Edition (CRC Press, New York, 2008).

3) K. O. Hill and G. Meltz: J. Light. Technol. 15 (1997) 1263.

4) A. Othonos and A. Kalli: Fiber Bragg Gratings (Artech House, Boston, 1999).

5) G. Laffont and G. Ferdinand: Meas. Sci. Technol. 12 (2001) 765.

6) R. Kasyhap: Fiber Bragg Gratings (Academic Press, San Diego, 1999).

7) T. Erdogan: J. Opt. Soc. Am. A 14 (1997) 1760.

8 A. Cusano, D. Paladino, A. Iadicicco, S. Campopiano, and C. Caucheteur: J. Res. Lett. Opt. 2009 Article ID 481010 (2009).

9) J. Rheims: Meas. Sci. Technol. 8 (1997) 601. 